Investigaciones Fenomenológicas, vol. Monográfico 6, e-ISSN: 1885-1088 en asociación con Escritos de Filosofía-Segunda Serie No 3, 2015, 289-311

\title{
EL CAMINO HACIA UNA ANTROPOLOGÍA TRASCENDENTAL
}

\section{The Way to the Transcendental AnTHROPOlOgY}

\author{
Javier San Martín \\ UNED \\ Madrid, España \\ jsan@fsof.uned.es
}

\begin{abstract}
Resumen: La obra de Julia V. Iribarne se basa, entre otros, en tres conceptos centrales, inter-subjetividad trascendental, ética trascendental y antropología trascendental. En este texto de homenaje a su persona y a su trayectoria intelectual, dado que nuestra autora se basa fundamentalmente en los inéditos de Husserl, aclararé, en primer lugar la posición del legado póstumo [Nachlass] husserliano en la obra de este. A continuación dedicaré un apartado a exponer el desarrollo de cada uno de esos conceptos a lo largo de su trayectoria, desde la intersubjetividad (apartado 2), pasando por la ética (apartado 3), hasta antropología trascendental (apartado 4).
\end{abstract}

Palabras clave: Iribarne, Julia V.; intersubjeti-vidad; ética fenomenológica; antropología filosófica

\begin{abstract}
Julia V. Iribarne's lifework rests, among others, on three central concepts, transcendental intersubjectivity, transcendental ethics and transcendental anthropology. In this text in tribute to her person and intellectual trajectory, and because Julia V. Iribarne's writings are founded basically on Husserl's Nachlass, I shall first clarify my conception about the relation of this Nachlass to his work. Next I will dedicate a section to explain the development of each of the concepts throughout her career, from intersubjectivity, (paragraph 2), to ethics (paragraph 3) and to transcendental anthropology (paragraph 4).
\end{abstract}

Key Words: Iribarne, Julia V.; intersubjectivity, phenomenological ethics; philosophical anthropology.

La obra de Julia Valentina Iribarne está íntimamente vinculada a la publicación de los manuscritos inéditos de Husserl. En efecto, su doctorado, llevado a cabo a partir de la publicación, en 1973, de los tomos XIII, XIV y XV de Husserliana, versó sobre la intersubjetividad en Husserl. Posteriormente, sus dos obras más significativas después de su trabajo de doctorado, La fenomenología 
como monadología y De la ética a la metafísica, toman en consideración principalmente los escritos husserlianos de la época de Friburgo, que como se sabe, permanecieron en su mayor parte inéditos. Todos estos trabajos se enmarcan en la nueva visión de Husserl que, desde el libro de Don Welton The New Husserl. A Critical Reader (2003), se conoce como "el nuevo Husserl". Sin embargo, la obra de la profesora argentina, quizás por dificultades de distribución, no ha sido aún muy difundida. En general, este número de nuestra revista debe servir para darle esa visibilidad. Por mi parte, me centraré en los tres temas básicos de la obra de Julia V. Iribarne. En el primer apartado comentaré la problemática de obra de Husserl en relación con su legado póstumo, lo que se conoce como su Nachlass ${ }^{1}$, dada la importancia de este en la obra de la profesora argentina. En los otros seguiré los tres temas centrales de la investigación de J. V. Iribarne, la intersubjetividad, la ética y la antropología, todos en un plano trascendental, aspecto que es el punto clave.

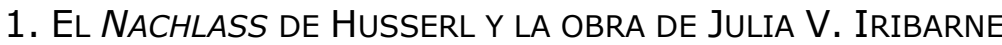

Según consta en la presentación que Roberto Walton hace de la trayectoria intelectual de Julia Iribarne para su ingreso en la Academia de Ciencias de Buenos Aires, Julia había obtenido el doctorado en 1988. Es justo el año en que la conocí, en el Congreso de Santiago de Compostela, que organizamos con Maria Teresa Tyminiecka. Mi impresión de Julia fue la de una señora elegante y prudente, a la que no sabía encuadrar académicamente porque, pareciéndome mayor que yo - aunque tampoco pude nunca asegurar en qué medida lo era-, me parecía más joven en la Academia, pues hasta esa fecha nada de ella había tenido oportunidad de leer. Sin embargo, el tema que trabajaba y que se publicó precisamente entonces coincidía a todas luces con mis propias preocupaciones. Sus dos tomos sobre la intersubjetividad me dieron una pista muy importante. Recuerdo que el organizador del Congreso en Santiago, Nel Rodríguez Rial, y yo tuvimos con M. Teresa Tyminiecka un almuerzo en el que salió el tema de los manuscritos de Husserl, sobre los que yo había basado en gran medida mi tesis doctoral, de 1972. La opinión de la Sra. Tyminiecka era que esos

\footnotetext{
${ }^{1}$ Se llama Nachlass en alemán a la totalidad de los inéditos de un autor a la hora de su muerte. En español sería el legado póstumo, la herencia, pues Nachlass literalmente significa "lo dejado después", que aquí, aplicado a los escritos, significa los inéditos y textos editados póstumamente.
} 
manuscritos no podían alterar el sentido de las obras publicadas por Husserl; que ni siquiera hubiera de tomárselos en serio, hasta, tal vez, ni siquiera existiría el derecho a hacerlos públicos puesto que su autor no lo había hecho. Mi escándalo fue mayúsculo, tanto más cuanto el mismo Husserl estaba convencido de que entre sus manuscritos había no sólo textos de trabajo sino verdaderas investigaciones consolidadas, "ciencias enteras" [ganze Wissenschaften], como con frecuencia los denominaba cuando alguien comentaba que aún faltaban por desarrollar partes de su obra, por ejemplo, la fenomenología genética, y él respondía con esas palabras señalando a los lugares donde guardaba sus manuscritos. Con ello se estaba indicando que no era verdad que no publicaba esos manuscritos por ser páginas impublicables, o porque no quisiera que vieran la luz. Más bien lo contrario, prefería trabajar en nuevas investigaciones a invertir en las tareas tediosas y que tanto tiempo exigen para ultimar y redondear los textos y, después, tener que seguirlos hasta su publicación.

En su momento le comenté a Julia V. Iribarne la opinión de M. Teresa Tyminiecka y evidentemente su escándalo fue también mayúsculo porque toda su obra se había basado principalmente en los póstumos de Husserl. Para mí, esa dificultad que planteaba M. Teresa Tyminiecka había sido una cuestión inexistente, nunca me planteé el tema, quizás porque, habiendo empezado a leer a Husserl desde las intuiciones de Merleau-Ponty, que se basaba en la lectura que había hecho de algunos textos póstumos de Husserl, y de la VI Meditación cartesiana de Fink, para mí el Nachlass del filósofo moravo era parte integrante de su obra. Por supuesto, lo mismo que para Julia V. Iribarne.

Y justo esa pertenencia de las publicaciones póstumas a la obra de Husserl es lo que se convirtió en el centro de la fenomenología de Julia Valentina Iribarne. Para describir qué significa eso, me basta con fijarme en la oposición entre el Husserl que se genera con la publicación de Ideas I, y el Husserl que emerge, principalmente, en la época de Friburgo. En Ideas I Husserl propone su método de la epojé y la reducción, por el cual el mundo es puesto entre paréntesis, para quedarnos con el mundo como correlato de mi experiencia, como noema de las múltiples noesis que constituyen la vida subjetiva. Desde esa idea, la fenomenología pura, como descripción del yo puro, es la filosofía primera, mientras que todos los análisis posteriores de lo que aparece en la experiencia serían temas de filosofía segunda. Ante todo las ontologías regionales que tratan de los diversos objetos de la experiencia, primero, la naturaleza; 
segundo, mi propio cuerpo, con su alma, constituyendo cuerpo y alma la persona humana, por supuesto, como parte del mundo; luego los otros, como partes también del mundo; y tercero, el reino del espíritu, la cultura, incluida aquí la ciencia. Si el estudio del humano es la antropología filosófica, que así es una ciencia regional sometida a la filosofía primera, también lo son el saber de los otros y, por supuesto, la ética, que regula los comportamientos del sujeto en relación consigo mismo y con los otros. Obviamente, también los temas relacionados con el principio y fin de la vida, o la inserción del propio sujeto trascendental en la historia, que son los grandes problemas metafísicos. La filosofía fenomenológica, que abarcaría todos estos saberes, de carácter regional, es, así, una filosofía segunda, respecto a la cual la fenomenología pura expuesta en Ideas I es la filosofía primera. Este esquema fue el usual, y el que podemos llamar ortodoxo, frente al cual, por otro lado, se revelaron muchos discípulos y alumnos de Husserl dando lugar a las nuevas orientaciones en la fenomenología, que terminaron por aparecer como heterodoxias, tanto que se popularizó el dicho de que la historia de la fenomenología es la de sus heterodoxias.

El principal problema vino, como se sabe, del giro trascendental que se produce en las Ideas $I$, a resultas de la práctica de la epojé y la reducción, entendidas ambas como una unidad metodológica por la cual el mundo de la actitud natural, el mundo en el que vivimos y actuamos, ese mundo descrito en el $\S 27$ de Ideas I, donde se dice que estoy en un mundo de cosas, pero en un mundo que, con la misma inmediatez de las cosas, se me da:

como un mundo de valores, mundo de bienes, mundo práctico. Sin necesidad de más, encuentro las cosas ante mí pertrechadas, así como con cualidades de cosas, también con caracteres de valor, como bellas y feas, como agradables y desagradables, como encantadoras y chocantes, etc. En forma inmediata hay cosas ahí como objetos de uso, la "mesa" con sus "libros", el "vaso", el "florero", el "piano", etc. También estos caracteres de valor y estos caracteres prácticos pertenecen constitutivamente a los objetos de "ahí adelante" en cuanto tales, vuélvame o no a ellos y a los objetos en general" (Hua III/1, 58; Zirión 137).

Pues bien, ese mundo, el mundo de las personas, en el que vivo, según se dirá en Ideas II, en actitud personalista, y que es en el que estamos y actuamos, se pone entre paréntesis, para dedicarnos al mundo que se da a la conciencia.

El que leía a Husserl desde las Ideas, e iba ampliando su conocimiento desde esta obra, entendiendo también Meditaciones cartesianas desde ahí, no te- 
nía otra posibilidad de comprenderlo entenderlo más que desde el esquema anterior, facilitando así el conocimiento de una fenomenología clara, en la que Husserl estaba relativamente encasillado como un idealista o un trascendentalista al estilo kantiano o neokantiano, en el que el sujeto trascendental es el yo constituyente del mundo, y todo el resto objetos constituidos en este mundo. Como la constitución, además, se hace de acuerdo a esquemas cognitivos, porque es muy menor lo publicado por Husserl en referencia a otro tipo de actos, el predominio de la teoría sobre la práctica parece total. Por otro lado, ese esquema hace de la fenomenología una filosofía escasamente apta para pensar un mundo convulso como el que surgió a partir de 1914 y que dio lugar al violento e inestable siglo XX. La fenomenología prevista por Husserl de modo sistemático en las Ideas I no parecía una filosofía adecuada al siglo, y por eso una vez llegados los nazis al poder, prácticamente desapareció de la escena filosófica, siendo sustituida por lo que fue conocido como las 'herejías' de la propia fenomenología husserliana, por ejemplo, en Alemania, la filosofía heideggeriana prolongada después con la hermenéutica gadameriana; y en Francia, la fenomenología francesa, que pronto derivaría en la filosofía deconstructivista, y epígonos, tales como las filosofías de Deleuze y otros parecidos.

Todas estas filosofías se basaban en el mantenimiento de aquella visión de Husserl prevista en el tomo primero de Ideas, de acuerdo a la cual el mundo ordinario de la vida, en el que viven o vivimos las personas, tiene que ser puesto entre paréntesis, por lo que no tendríamos que contar con él.

El problema fue que ya en la época de Friburgo ese esquema se había roto. Que los que estaban con Husserl en Friburgo, primero Landgrebe, luego Dorion Cairns y Fink, estaban siendo testigos de una fenomenología muy distinta, que no encajaba en el diáfano esquema de Ideas I. Y con la publicación sucesiva de los póstumos de Husserl se fue abriendo paso una nueva visión de este profundamente distinta de aquella primera y que convertía esa filosofía en una filosofía adecuada al mundo contemporáneo. $Y$ en esa tarea trabajó siempre Julia, desde su tesis doctoral sobre la intersubjetividad.

La amplia contribución de Julia Iribarne, entre otras muchas que en este monográfico se están poniendo de relieve, está en el uso que ella hace sin ningún prejuicio de tres conceptos o temáticas, cuyo sentido topológico quiero exponer en este trabajo. 
La primera se refiere a la posición de la intersubjetividad; de hecho, de esta arrancan las otras dos. No en vano hizo Julia Valentina Iribarne su tesis doctoral sobre la intersubjetividad. Roberto Walton lo señala muy bien en la mencionada presentación de nuestra autora en su admisión en la Academia de Ciencias de Buenos Aires, ya que se refiere al giro que Julia detecta con el tema de la Intersubjetividad: "El mérito de esta obra es haber puesto de relieve el gran giro efectuado por el pensamiento de Husserl, esto es, la localización de la intersubjetividad en el ámbito trascendental" (Walton, 2005: 5), por tanto, incorporar a la fenomenología la tesis de que la responsable de la constitución del mundo es la subjetividad trascendental pero en la medida en que es intersubjetividad trascendental. Si la reducción trascendental, que es el verdadero acceso a la fenomenología trascendental, es consecución del yo trascendental o subjetividad trascendental en el yo humano que soy yo, debe producirse el giro correspondiente para conseguir la intersubjetividad trascendental a través de una correspondiente reducción intersubjetiva. Si con la reducción conseguimos descubrir en el yo individual que somos cada uno su sentido trascendental - definición precisa de la reducción, como reconducción del ser humano a su sentido trascendental, por la cual también y simultáneamente el mundo es reconducido a su sentido trascendental como correlato de la subjetividad-, lo mismo debe ocurrir con la humanidad, el conjunto empírico de los humanos, que debe ser reconducido a su sentido trascendental. Este es el sentido preciso de reducción trascendental intersubjetiva, por la cual en los otros - dados bien como presencias reales, bien como ausentes o también como horizontes sociales, pero en todo caso siendo todos ellos de quienes tomo el sentido de mi identidad socialse descubre un sentido trascendental, al que queda reconducido el mundo como su correlato de realidad, de valoración, de acción y sedimentación de las instituciones de sentido que se efectúan en la historia, por lo que también es un mundo histórico.

Dicho así, además de no tener, aparentemente, esa frase sentido para la inmensa mayoría de personas ${ }^{2}$, parece algo de escasa relevancia, pero de he-

\footnotetext{
${ }^{2}$ Recuérdese lo que le dice Levy-Bruhl a A. Gurwitsch cuando lee la carta que le mandó Husserl el 11 de marzo de 1935: "Vgl. H. SPIEGELBERG, Scrap-Book (A. Gurwitsch): Levy-Bruhl, after receiving H.'s letter in 1935, showed it to Gurwitsch and asked: "Expliquez-moi, je n'en comprends rien." (Schuhmann, 1977: 459). Para una explicación de la carta, ver Edmund Husserl, "Carta a Lévy-Bruhl", traducción de J. San Martín, en revista ER, Revista de Filosofía, 19, año X, pp. 171-175 y de J. SAN MARTín "Husserl y la antropología cultural. Comentario a La carta de Husserl a Lévy-Bruhl", en revista ER, Revista de Filoso-
} 
cho es un punto en el que se rompe todo el esquema topológico bosquejado en Ideas I, lo cual, evidentemente, altera de raíz la fenomenología con la que se estaba operando hasta entonces. Por tanto, Julia V. Iribarne se sitúa, ya desde su tesis doctoral, en un Husserl al otro lado del Husserl tradicional, al que en mi libro La nueva imagen de Husserl (San Martín, 2015; ver también 2012) llamo el Husserl "convencional".

La segunda está también en relación con este punto. Porque en el esquema primero los otros era objetos en el mundo. Según el sentido del cambio, el giro en el tema de la intersubjetividad, también la ética tendrá que cambiar de topos, de ser una disciplina secundaria, de modo consecuente con el esquema previsto en Ideas I, al introducirse en el interior del sujeto trascendental el otro, no como otro individual sino como conjunto de los otros: la intersubjetividad. Con ello, mi comportamiento respecto de esta alteridad ya no es algo externo al yo puro, sino que le afecta, radicalmente, desde dentro. La ética se convierte en un saber trascendental. Hay una ética trascendental, que proviene del hecho de que llevamos a los otros dentro de cada uno de nosotros.

La tercera está en relación con este giro, y no es, topológica o arquitectónicamente, menos problemática que las dos primeras. En efecto, uno de los temas que más claro parece estar en el esquema husserliano inicial es que la fenomenología no es una antropología porque lucha de igual manera contra el psicologismo que contra el antropologismo, lo que significa despojar a estas ciencias o saberes de su pretensión de ser las ciencias primeras que sustituirían a las hasta entonces postuladas como tales, fundamentalmente la metafísica; o ya en la Modernidad, una filosofía del yo; o en el caso kantiano, una crítica de la razón pura. El sujeto puro, como sujeto garante de la verdad, no podría ser el humano resultado de azares históricos puramente contingentes, como es el desarrollo mismo de la evolución. La verdad y el bien no parecen ser de este mundo, sino de otro. Justo eso es lo que se querría indicar con la palabra 'trascendental'. El yo puro precisamente por ello no es el ser humano, que está lejos de ser puro en el sentido fenomenológico porque el cuerpo está sometido a mil azares; en él casi todo es radical facticidad y contingencia, desde el nacimiento hasta la muerte. Pues bien, con el tiempo, aunque en este caso ya más

fía, 19, año X, 1995, pp. 177-204. Actualmente en SAN MARTín, 2007. Lo que seguramente Levy-Bruhl no entiende de la carta es la referencia del mundo a la subjetividad, que es lo que significa ser correlato. 
al final de su vida, y justo después de haber pronunciado la famosa conferencia sobre "Fenomenología y antropología", en 1931, para rechazar las derivas de Heidegger y Scheler hacia una antropología filosófica -al margen de que se pudiera decir eso en el caso de Heidegger-, Husserl da un decidido giro y se plantea la relación con la antropología y la fenomenología en tonos profundamente distintos.

En un texto de esa época llega a hablar Husserl de una nueva antropología, una auténtica antropología frente a la anterior, y que no sería otra que la que tendríamos que llamar, y así la llamará Julia V. Iribarne, "antropología trascendental".

Los tres conceptos involucrados en esas temáticas, la intersubjetividad trascendental, la ética trascendental y la antropología trascendental creo que cubren en gran medida la aportación de Julia a la fenomenología, entendiendo a Husserl desde la totalidad de su obra, fundamentalmente desde su Nachlass. Voy a procurar ampliar el sentido de los tres términos, que podrían servir también para dar contenido a lo que en la actualidad se llama el "nuevo" Husserl.

\section{EL GIRO: LA LOCALIZACIÓN DE LA INTERSUBJETIVIDAD EN EL ÁMBITO TRASCENDENTAL}

Todos sabemos que el tema de la intersubjetividad fue absolutamente decisivo en la trayectoria y recepción futura de la fenomenología de Husserl. Para él mismo, tal como lo hemos terminado sabiendo, fue un verdadero caballo de batalla, porque, fundada la fenomenología, al estilo cartesiano, en la evidencia del ego cogito, el solipsismo que ahí acecha no puede dejar de perturbar. Una condición imprescindible para convertir la fenomenología en algo a tener en cuenta y con futuro era resolver cómo, de la certeza del yo como punto nuclear de la fundamentación, se podía pasar a una certeza compartida, es decir, cómo superar el solipsismo epistemológico.

La fenomenología, como filosofía primera, tiene, ante todo, la función de ofrecer a las ciencias un fundamento que pudiera asentarlas en un terreno firme, y así prepararlas para superar las tentaciones del escepticismo que las cuestiona; para ello empieza por la reducción fenomenológica, en la que descubro y convierto en tema de análisis mi vida, que es trascendental por ser condición de posibilidad de toda aparición, cognitiva y valorativa, así como de toda decisión práctica y el lugar en el que se tiene que dar aquella fundamentación. 
Pues bien, sólo valdrá esa vida trascendental como tal fundamento, si no sólo es válida para mí, sino si es válida para todos. Pero justo por el modo en que Husserl llevó a cabo la reducción, parece que los otros han desaparecido de mi horizonte. De ahí la importancia de hacer ver que la vida trascendental no soy yo sino que, siendo yo, somos en realidad todos. La vida trascendental es intersubjetiva, es un sujeto compuesto por todos los sujetos. Por tanto, técnicamente, la reducción trascendental, que me tenía que servir para hacer visible el yo trascendental, mi vida trascendental, debe servirme también para lograr la intersubjetividad, es decir, para comprender mi yo trascendental como intersubjetividad, que para cumplir la función asignada debe ser también trascendental.

Pues bien, aquí hay dos momentos funcionalmente distintos: el primero es cómo percibo al otro, que es un tema propio de un análisis constitutivo como el de cualquier otra cosa, tal como Husserl los realiza, por ejemplo, en Ideas II respecto al cuerpo y a la cultura, esta en tanto que "objetos espiritualizados". Pero el segundo punto, mucho más relevante, es en qué medida esa experiencia del otro equivale a la aparición o descubrimiento de la intersubjetividad trascendental. Para ello, el otro ha de ser descubierto como otro trascendental, y eso es la ampliación de la reducción fenomenológica trascendental a una reducción fenomenológica intersubjetiva. $Y$ este es el punto clave, un punto que no es fácil de percibir, siendo fundamental. En primer lugar, en la obra publicada de Husserl nunca se habló de este extremo. Más aún, se tomó la "V Meditación cartesiana" como un esfuerzo, por otro lado, baldío (ver Iribarne, 1987a: 72), para salir del ego aislado al otro, sin tomar nota de que ahí se trata de esa ampliación de la reducción trascendental a una reducción intersubjetiva.

Puede ser que en este punto Julia no haya sido lo suficientemente osada para hablar sin precaución ninguna, a tumba abierta, de "reducción intersubjetiva". El principal mecanismo para esa reducción intersubjetiva es la doble reducción, de la que se empieza a hablar en la Grundproblemevorlesung, de lo que Julia Iribarne da fe en varias ocasiones ${ }^{3}$. Pero no me consta que llegue a definir esa doble reducción como el modo en que Husserl lleva a cabo la reducción intersubjetiva. Es cierto que Husserl no es pródigo en esa denominación. Y segundo, es Fink quien nos indica que la V Meditación cartesiana tenía ese objetivo, cuando dice que la V Meditación supone la "culminación de la reducción

\footnotetext{
${ }^{3}$ El tema sale de modo reiterado. Ver IrIBARNE, 1987b: 45, 47, 51, 53-57, 65, etc.
} 
egológica hacia la reducción intersubjetiva implícita en ella misma [en la anterior, en la egológica]"4 ${ }^{\prime 4}$ Cierto que la tesis de Julia Iribarne se concluyó antes de la edición de estos textos de Fink, que se publicaron a finales del otoño de 1988; pero no vuelve después sobre esa denominación, por más que en la edición alemana de las Meditaciones cartesianas Husserl hablara, él mismo, de esa "reducción intersubjetiva", cuyo objetivo no es otro que mostrar la trascendentalidad de los otros, esa caracterización del otro que para Julia Iribarne será decisiva de todo punto de vista: que llevamos a los otros en nosotros mismos; porque, en definitiva, la reducción intersubjetiva tiene que hacerse a partir de mis experiencias de los otros, mostrando que incluyen el poner al otro como otro trascendental.

En el magnífico prólogo al libro de J. Iribarne La fenomenología como monadología Roberto Walton lo explica con toda claridad:

Así, la legitimación de la condición trascendental del otro no reside en su propia reducción trascendental, que bien podría no ser efectuada por él, sino en esa reducción mía "en" el otro que es posible porque la empatía admite también el procedimiento de la doble reducción. Con otras palabras, la explicitación de las implicaciones intencionales de la propia vida del ego nos lleva más allá de ella, Y pone de manifiesto un mayor alcance de la vida trascendental al convertir la reducción egológica en una reducción intersubjetiva (Walton, 2002: 13).

Ahí está explicitado de modo perfecto el sentido de la teoría trascendental del otro, de la que Husserl nos había dicho en una anotación puesta debajo del texto de las Meditaciones cartesianas, que de la reducción intersubjetiva, en París, sólo había hecho "indicaciones verbales superficiales", pero "de modo sistemático desarrolladas más próximamente en Estrasburgo" ${ }^{5}$. Toda la tarea posterior de Julia Iribarne se basa en estos descubrimientos de su tesis doctoral, aunque es muy posible también que quizás nunca dejara, en alguna manera, de estar vinculada con una concepción de la reducción como epojé antes que como reconducción, condición para hablar sin problemas de una "reducción

\footnotetext{
4 "Vollendung der egologischen Reduktion zu der in ihr selbst implizierten intersubjektiven Reduktion", FINK, 1988b: 250.

${ }^{5}$ Hua I, 236, a 39, 30: "über intersubjektive Reduktion nur flüchtige mündliche Andeutungen, systematisch näher ausgeführt in Straßburg". Se cita esta anotación en Hua I, Einleitung, XXIV, de Strasser. Estas indicaciones fueron para mí decisivas para poder responder a la pregunta que me hacía sobre como pasar del yo al otro, que se me planteó, en 1969, con la lectura de Merleau-Ponty. Por eso la tercera parte de mi tesis doctoral sobre la reducción se tituló directamente "La reducción intersubjetiva". Un error en la gestión de mi profesión de investigador dejó esa tercera parte sin publicar, ni siquiera publiqué la reelaboración que de ella tenía ya terminada a finales de los setenta, con lo que esa faceta, muy novedosa, de mi trabajo de doctorado de 1972 quedó desconocida.
} 
intersubjetiva". Esta es la reconducción de los otros mundanos a su sentido trascendental. Por eso, si entiendo la reducción como epojé, se hace difícil esta descripción. En efecto, en el primer capítulo de La fenomenología como Monadología, que publica el que posiblemente es el primer trabajo amplio de Julia V. Iribarne sobre la fenomenología, pues es de 1983, se dice de la reducción: "«Reducir» quiere decir desconectar, poner en suspenso ciertas afirmaciones y en primer lugar la que concierne a la existencia del «mundo» de que la conciencia da testimonio. El fenomenólogo lleva a cabo la epojé del juicio de existencia" (Iribarne, 2002: 46). Un poco más adelante se cita la frase de Meditaciones cartesianas en que aparece la palabra "reconduce", que traduce la alemana "zurückleitet". La epojé en la medida en que reconduce 6 a la subjetividad trascendental, es reducción, y en la medida en que reconduzca a la intersubjetividad trascendental, será reducción intersubjetiva.

\section{EL LUgAR tRASCENDENTAL DE LA ÉTICA EN HUSSERL}

He comentado las dificultades de distribución de la obra de Julia V. Iribarne. En 2008 participé en el Congreso Internacional de la Sociedad Hispanoamérica de Filosofía que tuvo lugar en Medellín, que se dedicó de manera especial a homenajear a Guillermo Hoyos Vázquez, enfermo ya por entonces y que acababa de salir de una fuerte intervención quirúrgica. Con ese motivose publicó el libro de homenaje al amigo y profesor La responsabilidad del pensar ${ }^{7}$. Tuve la suerte de adquirir el libro y encontrarme en él con dos textos magníficos, el de Roberto Walton y el de Julia V. Iribarne, que ocupan nada menos que ochenta y cinco páginas. Más de cuarenta cada uno. El artículo de J. Iribarne me impactó mucho por lo que luego diré. El de R. Walton es un excelente estudio, como todos los suyos, sobre el lugar de la ética en Husserl, que, por supuesto, complementa al siguiente.

\footnotetext{
${ }^{6}$ Julia cita la traducción del argentino Mario A. Presas. En la traducción de Gaos, "zurückleitet", se traduce como "retrotrae" (HuSSERL, 2004: 62), con lo que se pierde el sentido de ser una de las dos palabras alemanas para reducción, Zurückführung y Zurückleitung. Por otro lado, al final de la frase: "La epojé en la medida en que reconduce [retrotrae, en Gaos] a la subjetividad trascendental", dice Gaos que "puede llamarse la reducción fenomenológico-trascendental". Ese "puede" no existe en alemán. El matiz es muy importante. También la traducción de Presas comete un error, pues el "ámbito trascendental" es un invento de Presas, pues en alemán se refiere a "der natürliche Seinsboden", que ha mencionado en la frase anterior, "la base natural del ser" -y no "la base del ser natural". Mirar las traducciones de textos alemanes de fenomenología resulta muchas veces un llorar por nuestro pésimo nivel.

${ }^{7}$ Alfredo ROCHA DE LA TORRE (ed.), La responsabilidad del pensar, Barranquilla (Colombia): Ediciones Uninorte, 2008.
} 
La razón del impacto a que aludo, es que el trabajo de J. Iribarne contiene un análisis de los aspectos esenciales a los que Husserl dedicó su atención en el terreno de la fenomenología genética, esto es, la historia de la subjetividad trascendental que se va haciendo desde los instintos, la síntesis pasiva y la comunidad del amor. La autora insiste en que el carácter novedoso de la fenomenología trascendental husserliana respecto de otras filosofías trascendentales reside en su concepción de los instintos trascendentales capaces de instituir un sistema de aprehensión del mundo y fundar el vínculo intersubjetivo, temas sobre los que volveré inmediatamente. Pero lo que quiero decir ahora mismo es que solo mucho después supe que la contribución de Julia en ese libro era el capítulo IX de su otro gran libro De la ética a la metafísica, publicado el mismo año 2008 -aunque en la edición ponga 2007-, pero no muy distribuido ${ }^{8}$. Supongo que la profesora Iribarne tampoco hizo mucho por su distribución, quizás debido a un incidente de salud, pero el hecho es que esta magnífica obra quizás no ha recibido aún la atención que merece.

Llegado un momento ya muy avanzado de su teoría de la intersubjetividad en Husserl, Julia Iribarne se hace una pregunta decisiva: "El bosquejo de la teoría podría introducirse con un planteo preliminar: ¿Cuál es el verdadero problema para Husserl, el problema fundamental de la fenomenología: la pregunta por el otro o la pregunta por el mundo?" (1987b: 124). Está claro que la segunda tiene preferencia, pero igualmente es patente que sólo puede responderse por la primera, y en esa respuesta aparecen las implicaciones prácticas de la fenomenología, que terminan por desvelar que lo que mueve a la fenomenología no es sino la creación de una humanidad auténtica, cuyo núcleo yo diría que es lo que Julia Iribarne Ilama en su momento "la responsabilidad universal por el otro" (Iribarne, 2009: 29). La fórmula del ethos filosófico, el compromiso del filósofo con la creación de una "humanidad auténtica" no la teníamos hasta conocer las Londoner Vorträge, pero esa humanidad auténtica no es otra que la noción de monadología propuesta por Husserl ya muy pronto. El problema del mundo entonces no era más que un motivo para cumplir lo que, con Arion L. Kelkel, me gusta llamar la "vocación antropológica de la fenomenología" y, en

\footnotetext{
${ }^{8}$ Puedo decir que solo en una de las bibliotecas universitarias españolas se encuentra este libro, casualmente una universidad en la que enseña filosofía un profesor argentino.
} 
esta, la ética tiene un lugar privilegiado, siendo además peculiar de la etapa de Friburgo, o del Husserl de después de la Guerra del 14.

Ya en 1990 escribe Julia Iribarne un decisivo artículo, al que quizás no se le prestó excesiva importancia. De haberlo hecho, deberíamos haberlo citado numerosas veces, lo que no ha sido el caso. Cierto que el lugar de publicación no tenía apenas difusión, la revista de Cuadernos de Ética, por entonces relativamente desconocida. El artículo ha sido recogido como capítulo XVII de La fenomenología como monadología, y efectivamente ahí se ve el camino de la fenomenología en la visión de Julia Iribarne, desde la intersubjetividad pasando por la ética a la antropología, todas por supuesto en un plano trascendental. En ese artículo, que ahora considero decisivo, hasta el título, "Interpretación ética de la 'interpretación' fenomenológica", puede resultar poco esclarecedor, pues en él se trata nada menos que de "una nueva fundamentación trascendental de la ética", como dice a poco de iniciarlo (Iribarne, 1990: 33; 2002: 289). La razón del título está seguramente en un intento de hacer parecer la intersubjetividad de Husserl como un "tema hermenéutico". Hay que tener en cuenta que la Sociedad Argentina de Fenomenología lo es también de Hermenéutica, habiendo encontrado, principalmente, Paul Ricoeur un gran eco en Argentina, sobre todo a través de Mari France Begué. En este contexto el artículo de J. Iribarne es un intento de mostrar que en el núcleo central de la fenomenología, es decir, en la intersubjetividad constituyente, hay una interpretación. Por tanto la fenomenología está de lleno en el terreno de la hermenéutica. Pero eso evidentemente es una cuestión secundaria. Porque lo importante es que esa "interpretación", que es ni más ni menos que la experiencia del otro - J. Iribarne la llama la interpretación originaria (1990: 36 ss.; 2002: 293 ss.) - impone una moral. Y en la medida en que la intersubjetividad trascendental está en el núcleo de la fenomenología, la ética también lo está. Ese es el tema angular del artículo más allá de un título contemporizador. En él se pretende mostrar cómo desde la teoría de la intersubjetividad se llega a esa responsabilidad para con el otro, que es el fundamento de la moral.

Para ello, toma como punto de partida "La paridad de los yo según las operaciones que los constituyen" (1990: 37; 2002: 294). La paridad implica una repetición, es decir, el poner al otro con estructuras semejantes a las mías. Aquí aporta una cita muy importante de Husserl: "El otro es mi 'repetición' intencional y esto no sólo quiere decir que yo lo tengo a él como repetición (ac- 
tual o posible), sino también que él [...] también me tiene a mí como su repetición intencional" (Hua XV, 489). Esta repetición, que puede reiterarse hasta el infinito, funda la posibilidad de "constitución de un universo monádico, de mónadas en relación de paridad y de esencia igual [...]" (Hua XV: 636; Iribarne, 1990: 40; 2002: 298). Se quiere decir que, en mi experiencia del otro, este es puesto exactamente como yo, pero también como otro porque su vida le pertenece por el principio de que a las vivencias de cada conciencia sólo tiene acceso cada uno, pues el yo no admite plural (1900: 42; 2002: 300). Pero todo esto lleva a Julia a proponer que la igualdad trascendental que se corresponde con el modo de ser de la experiencia del otro equivaldría a algo así como a la igualdad nouménica kantiana, que es el sentido de lo que quiere llamar la "nueva fundamentación trascendental de la ética" $(43 ; 301)^{9}$, porque "el sentido reiterado de todas" las operaciones que acaba de citar, descubiertas en la experiencia del otro, "nos permite decir que en la igualdad en ellas configurada reside el fundamento trascendental de la ética" $(44 ; 302)$. Eso le permite terminar con una cita, que clarifica todo su ensayo, y que es de su compatriota Antonio Aguirre:

El Otro es también un yo como yo y posee en consecuencia todos los derechos implícitos en el concepto de yo, vale decir, todos los que yo tengo. Porque lo constituyo en la repetición, no sólo no lo privo de nada, sino que mediante la constitución le otorgo todo. Mediante la visión en la estructura de repetición de la percepción del Otro me despojo de todos mis posibles privilegios. $(45 ; 303)^{10}$.

\section{PARA UNA ANTROPOLOGÍA TRASCENDENTAL}

Y con esto llegamos ya al final de nuestro recorrido. La intersubjetividad es trascendental, y por eso implica unos deberes de unos respecto a los otros, y en concreto mi deber y mi responsabilidad para con los otros, por tanto, la ética no es algo regional que se debata en el terreno de lo constituido -en aquel topos de la filosofía segunda-, sino en el terreno constituyente, en el terreno de lo trascendental, de la filosofía primera. Pero en todo este proceso no ha dejado de haber un giro radical de la fenomenología. Porque cabría preguntar si el esquema previo de la filosofía primera y segunda sigue en vigor.

\footnotetext{
${ }^{9}$ En De la ética a la metafísica, explora J. Iribarne este punto de la mano de H. Rainer Sepp, donde se habla de en qué medida la ética es reasumida por la filosofía trascendental (Iribarne, 2008: 157ss).

${ }^{10}$ La cita de Aguirre es de Die Phänomenologie Husserls im Licht ihrer gegenwärtigen Interpretation und Kritik, 1982, p. 42.
} 
Por mor de la brevedad, no dedicaré todo el espacio que debería a este punto, que es en el que estoy más implicado, ya que casi toda mi tarea profesional está en relación con él. Solo voy a indicar algunos de los pasos que dio Julia V. Iribarne en una dirección que justo en estos momentos se está revalorizando en la fenomenología después de haber sido obviada, podríamos decir, hasta con displicencia.

Una ética consciente de sus implicaciones debe conducir a otro tema capital: la metafísica. En De la ética a la metafísica (2007), el esfuerzo se encamina a desarrollar una antropología trascendental, sobre todo porque en el fondo los problemas fundamentales de la metafísica son los problemas de nuestra estancia en el mundo a partir de nuestro nacimiento y hasta la muerte, la presencia en el mundo de cada uno de nosotros como humanos. Los problemas metafísicos, en Husserl, se refieren a las preguntas últimas de la vida humana. Tratar de plantear esas preguntas de modo correcto, y tratar de responderlas, asumiendo con una actitud humilde la dificultad de la tarea, ese es el sentido último de la filosofía. Pero eso significa que son preguntas antropológicas. La posición, entonces, de la antropología en relación con la fenomenología es lo que hay que dilucidar previamente porque no parece que al principio le gustara a Husserl confundir fenomenología y antropología. Más bien lo contrario, todo su esfuerzo metodológico estuvo dirigido a salvaguardar la fenomenología de la antropología. Sobre todo cuando percibió que, ya de jubilado en Friburgo, había una tendencia creciente a ver en la antropología filosófica la fórmula básica de la filosofía.

No es este un tema que aparezca pronto en Julia Iribarne. De hecho en sus dos primeros libros, sobre la intersubjetividad y la monadología, no es un concepto que aparezca. Tan sólo lo hace en el libro de 2007. En este, como ya he anunciado, ese tema se explora en el capítulo IX, pero se había hecho notar con anterioridad. Por otro lado, ya en 1990, en el artículo que he citado en las páginas anteriores y que luego será capítulo XVII de La fenomenología como monadología recoge una frase de Husserl, de las lecciones de 1927, sobre naturaleza y espíritu, que nos debería haber dado una pista sobre esta orientación de la fenomenología. En efecto, Husserl dice - y lo recogió Iso Kern, de quien lo 
toma nuestra autora ${ }^{11}$ - que "el carácter fundamental de la fenomenología es ser filosofía científica de la vida"12.

Otro texto importante es el que cita de la conferencia "Fenomenología y antropología", donde dice Husserl que el "Auténtico análisis de la conciencia es, por así decirlo, hermenéutica de la vida consciente que se refiere (vermeinen: que intenciona) a un ser que siempre es (idéntico), un ser que se constituye intencionalmente en multiplicidades de conciencia" (Hua. XXVII, 177). Julia Iribarne, en aras de ver que en Husserl sale la palabra 'hermenéutica', cita solo parcialmente la frase: "Auténtico análisis de la conciencia es, por así decir, hermenéutica de la vida de conciencia" (ib.). Este texto como se sabe es 1931. Pero el 26 de diciembre de 1927 le escribe Husserl a Mahnke una larga carta en la que le agradece la recensión que este había hecho del tomo VII de las Gesammelte Werke de Dilthey, que le había enviado a Husserl, y en la que se refiere a este, para terminar pidiendo una síntesis entre Husserl y Dilthey. Husserl le dice que, a partir de 1905, su filosofía ya es esa síntesis, que se produjo a raíz de su conversación con Dilthey en Berlín. Añade que le impresionó mucho que Dilthey considerara la filosofía de las Investigaciones lógicas idéntica a una psicología como ciencia humana, y que la pusiera en el contexto de su meta vital de llevar a cabo "una fundamentación filosófica de las ciencias humanas", esto "me causó una fuerte impresión", dice Husserl; y a continuación explica que desde entonces se dedicó a exponer los problemas de una "geistewissenschafliche Phänomenologie", actualmente diríamos: de una fenomenología como ciencia humana; que Ideas II, diseñada en 1912, "trata estos problemas ya de modo extenso", y que incluso de Ideas I se deduce "que para mí la fenomenología no es sino ciencia universal y 'absoluta' del espíritu". (Husserl, Briefwechsel, III, 460).

\footnotetext{
${ }^{11}$ Del prólogo a Hua XV, p. XLVII.

12 IRIBARNE, 1990: 34; 2002: 291. Dice Husserl, y es un texto muy interesante, que adelanta muchas cosas: "El carácter fundamental de la fenomenología es por tanto [el ser] filosofía científica de la vida, es ciencia, no bajo presupuestos y sobre la base de las ciencias ya dadas, sino ciencia radical que tiene como tema originario científico la vida concreta universal y su mundo de la vida [Lebenswelt], el mundo concreto real; que partiendo desde ahí y creando de modo puro desde la intuición más concreta lleva la tipología estructural de ese mundo a conceptos rigurosos y que en todo caso deben ser comprobados, y que desde ahí consigue de modo sistemático la conceptualidad fundamental que debe servir a todas las ciencias, del mismo modo que por otro lado tal ciencia muestra que todas las ciencias posibles solo pueden tener sentido en relación con las estructuras originarias de la realidad de la vida." Hua XXXII, 241 [org. de 1927]. Así, en este texto se adelanta la problemática del Lebenswelt, y la función epistemológica de la fenomenología en relación con las ciencias humanas, y todo ello de acuerdo al texto de la carta a Mahnke, que cito después.
} 
La fenomenología se presenta, pues, como la ciencia a priori para las ciencias humanas, para una fundamentación de estas, como se dice en el texto de las lecciones sobre Naturaleza y espíritu. Justo en ese momento también Ortega escribía el artículo "La 'Filosofia de la Historia' de Hegel y la historiología" (Ortega, 1928), en El que propone un análisis de la vida humana como una ciencia a priori para la ciencia histórica, exactamente lo que se encontró que había hecho Heidegger en Ser y tiempo, que pudo leer precisamente cuando acababa de escribir ese artículo.

Puestos a entender la fenomenología como ciencia hermenéutica de la vida consciente, o filosofía científica de la vida, es obvio que la pregunta por la relación de ese saber con la antropología filosófica que ya se estaba haciendo en Alemania tenía que surgir de inmediato. Y Husserl lo afrontó. También lo afronta Julia V. Iribarne, y aunque hasta el cap. IX no se toca el tema, nos salen algunos apuntes que habría que seguir para abordar el asunto a fondo, cosa que aquí no puedo hacer. En efecto, en el texto se deja caer algo así como la "concepción antropológica" de Husserl (Iribarne, 2007: 136), que no es la que podría surgir de Ideas II, donde el ser humano aparece como un objeto constituido en la subjetividad trascendental, pues en el caso de J. Iribarne está en relación con la ética, más en concreto con la personalidad ética, que se desenvuelve en un terreno trascendental. Habla igualmente de la "ontología antropológica" de Melle, aunque no hay referencia a ella tal como la concebiría Melle como "punto de partida" (Iribarne, 2007: 161). En todo caso, se está hablando de esa "ontología antropológica" o "concepción antropológica" de Husserl como de los análisis necesarios para comprender la ética husserliana, tal como éste la expone, por ejemplo, en los artículos sobre Renovación, donde la vida humana aparece como definida por la tendencia a la autoconservación y al cumplimiento de sus objetivos, entre los cuales el ideal ético es el fundamental.

Ya sabemos que la ética se enraíza en el terreno trascendental, por tanto teníamos que llegar a plantear la relación de la fenomenología con la antropología, cosa que hace Julia V. Iribarne de la mano de Wolfgang Orth (Orth, 1977) y del artículo que yo mismo escribí al final de mi estancia en Friburgo, en 1989, y que fue publicado en 1991 (San Martín, 1991). El estudio de ambos le lleva a postular una "antropología trascendental". Para ello toma como punto de partida la frase de Husserl, en que se basaría Orth, de que "todo ser humano 
lleva en sí un yo trascendental" (Iribarne, 2007: 179). Un poco más adelante nos habla de esa idea de antropología trascendental:

El ser humano mismo es un sistema de interacción en el sentido de una multiplicidad, que él tiene como a presentificar -en una síntesis identificante característica que se denomina persona-. Las expresiones sobre los comportamientos básicos antropológicos aparecen idénticas a lo expuesto acerca de las condiciones trascendentales de constitución, lo cual nos acerca al concepto de antropología trascendental". (ob. cit., 185).

El segundo momento en que sale la antropología trascendental es en relación, primero, con la idea de que las ciencias humanas o, como dice Husserl, lo empírico está sometido a una racionalización especial: "el juicio normativo según normas universales, que pertenecen a la esencia apriórica de la humanidad racional" (Iribarne, 2007: 186). La "humanidad racional" ha sido traducido por Agustín Serrano de Haro como "la condición humana 'racional'" (Husserl, 2002: $5)$, lo que permite entender mejor la intención de Husserl. La antropología trascendental incluye la elaboración de esa ciencia apriórica de la condición humana, que incluye la ética. $Y$ después de referirse Julia $V$. Iribarne al final de la conferencia "Fenomenología y antropología", que termina de un modo altamente paradójico, merece la pena citar el texto entero de J. V. Iribarne:

Después de los argumentos de E W. Orth y de J. San Martín, este texto de Husserl viene a proporcionar las respuestas que buscábamos. Habíamos dejado en claro que la ética, una vez superada la etapa en que como ontología racional se hallaba en el mismo plano que todas las demás ciencias, como ciencia universal tiene prioridad sistemática respecto de ellas. Por otro lado, la relación fenomenología y antropología sufre un proceso semejante: después de diferenciarse la antropología de la fenomenología, por no haber abandonado la primera la actitud natural, terminan por coincidir una con otra, en la medida en que la antropología reconoce su carácter intencional trascendental.

Resta aún ubicar la ética respecto de esta última relación; a este respecto el final de nuestro estudio formula preguntas. Dado que la fenomenología es la ciencia trascendental de la humanidad racional y, en esa medida, antropología trascendental, la ética como tendencia teleológica de la razón concierne a un ámbito de la misma. (Iribarne, 2007: 187)

Así, la ética quedaría subsumida en la antropología trascendental, siempre que ambas se entiendan en sentido trascendental, además, este es el sentido 
en que J. V. Iribarne las trata en este libro, porque ambas quedan subsumidas en la metafísica (ob. cit., 200) ${ }^{13}$.

Por fin, el último capítulo, titulado poética y filosóficamente "Éticamente habita el hombre el mundo" (ob. cit., 227 ss.) quiere exponer "las líneas principales de la antropología trascendental de Husserl", lo que hará en el número 3 del capítulo XII, donde se ofrece "una síntesis del pensamiento antropológico trascendental de Husserl", y para ello nos pone una frase que pudiera resultarnos problemática:

La consideración antropológica del ser humano es una consideración del ser humano como persona que está en el mundo y que se "refiere" al mundo como sujeto de la intencionalidad, (...). Sin duda el ser humano siempre es mentado como sujeto de su cuerpo vivido, como teniendo siempre un cuerpo vivido, como concorde con él (...) el ser en el mundo es mencionado exclusivamente de manera antropológica como referencia intencional de los sujetos entre sí y con el resto del mundo (Hua XV, 481).

El problema está en si esa "consideración antropológica del ser humano" es o no trascendental. De acuerdo a lo que comenta Julia Iribarne, se trataría, no tanto de la mera persona, como se dice en este texto, sino de la persona trascendental, de la que hablaba Husserl en 1930 (Hua XXXIV, 200), un texto publicado ya en 2002, pero que nuestra autora no cita a pesar de que en esas páginas se habla de la auténtica antropología. De todas maneras, en este bosquejo de antropología trascendental Julia V. Iribarne recupera los desarrollos anteriores sobre el ser humano: desde el hecho de hablar de una persona encarnada para la cual el cuerpo vivido es condición de posibilidad de su ser persona, hasta el ser de un modo que se va haciendo desde un horizonte instintivo, que lo lleva tanto a una situación intersubjetiva como hasta el uso de la racionalidad; un ser inserto en el mundo desde el vientre materno, y dotado de una tendencia hacia el cumplimiento de metas y fines, que actúa de modo motivado. $Y$ que por llevar a los otros en cada uno, tenemos una responsabilidad para con los otros, por lo que nuestro modo de habitar en el mundo es un modo ético de habitar.

\footnotetext{
${ }^{13}$ No quiero dejar de reiterar la inmensa influencia que en esta trayectoria de Julia V. Iribarne, de la intersubjetividad a la ética, y de esta a la metafísica por la mediación de la antropología filosófica, ha tenido la obra de Hans Rainer Sepp, cuya presencia en este libro de Julia Iribarne es constante. Las obras fundamentales de H. R. Sepp más tenidas en cuenta son su libro de 1997 y el artículo sobre Renovación (SEPP, 1994).
} 
Como colofón de este modesto ensayo que dedico a Julia V. Iribarne, quiero añadir un pequeño comentario biográfico sobre nuestra queridísima amiga. El caso de Julia V. Iribarne desde una perspectiva biográfica es atípico, pero, sin duda, absolutamente ejemplar. Como nos cuenta Roberto Walton en la necrológica -solo en ese momento he sabido yo su edad-, Julia había nacido en 1929, terminó su licenciatura en 1975, por tanto, a los 46 años, después de haber fundado con éxito una familia. Presentó su tesis de doctorado en 1988, momento en que la conocí, como una señora muy agradable y prudente, de edad indefinida, académicamente más joven que yo, pero que me parecía, por su edad, mayor que yo. A partir de su licenciatura, sin urgencias académicas, pudo dedicarse libremente a ampliar su investigación, visitar numerosas veces los archivos de Husserl, impartir docencia, cursos y conferencias, por invitación, en varias universidades, e ir publicando libros y trabajos de investigación con un ritmo continuo hasta su fallecimiento en 2014, dejando tras de sí una obra muy rica, que aún no ha sido apreciada en todo lo que vale. Cierto que se han dado problemas en la distribución de sus libros, pero, igualmente, me temo que con ella ha sido eficaz esa tendencia tan propia nuestra de minusvalorar lo escrito en nuestra lengua, porque, como podemos ver en este monográfico, esta obra es de una gran altura y radicalmente ajustada al momento interpretativo de la obra de Husserl, precisamente en la dirección en que este se está mostrando fecundo en el mundo contemporáneo, por lo que creo que tendremos que volver de modo reiterado y creciente a la obra filosófica de Julia Valentina Iribarne.

\section{BIBLIOGRAFÍA}

Aguirre, Antonio (1982), Die Phänomenologie Husserls im Licht ihrer gegenwärtigen Interpretation und Kritik, Darmstadt: Wissenschaftliche Buchgesellschaft.

FINK, Eugen (1988), VI. Cartesianische Meditation, Teil II, Ergänzungsband, ed. por Guy van KERCKHOVEN (Husserliana Dokumente, Bd 2/2), Dordrecht: Kluwer Academic Publishers.

HuSSERL, E. (1950-2014), Gesammelte Werke, La Haya, Dordrecht: M. Nijhoff, Kluwer, Springer. Se refieren los tomos citados con la abreviatura Hua. y el tomo en cifras romanas. 
- Husserliana I: Cartesianische Meditationen und Pariser Vorträge, , ed. e introd. de S. StRASSER, M. Nijhoff, La Haya, ${ }^{2} 1973$.

- Husserliana III/1: Ideen zu einer reinen Phänomenologie und phänomenologischen Philosophie. Erstes Buch: Allgemeine Einführung in die reine Phänomenologie, ed. de K. Schuhmann, La Haya: M. Nijhoff, 1976; trad. española de J. GAOS, Ideas relativas a una fenomenología pura y a una filosofía fenomenológica, Libro primero: Introducción general a la fenomenología pura, FCE, 1949, ${ }^{2} 1962$; nueva edición y refundición integral de la trad. por A. Zirión, México: FCE.

- Husserliana XV: Zur Phänomenologie der Intersubjektivität. Texte aus dem Nachlass. Dritter Teil: 1929-1935, ed. de I. KERN, La Haya: M. Nijhoff, 1973.

- Husserliana XXVII: Aufsätze und Vorträge 1922-1937, ed. de Th. NENON y H. R. SEPP, Dordrecht: Kluwer Academic Publisher. 1989.

- Husserliana XXXII: Natur und Geist. Vorlesungen Sommersemester 1927, ed. por Michael Weiler, Dordrecht: Springer, 2001.

- Husserliana XXXIV: Zur phänomenologischen Reduktion. Texte aus dem Nachlass (1926-1935), ed. de S. LufT, Dordrecht: Kluwer Academic Publisher, 2002.

Husserl Ed, Briefwechsel. III. Die Münchner Schule. Ed. de K. Schuhmann con la colaboración de E. SchummanN. Husserliana Dokumente III/ Band III, Dordrecht: Kluwer.

- (1979), Meditaciones cartesianas. Introducción, traducción y notas de M. Antonio Presas. Madrid: Ediciones Paulinas.

- (2002), Renovación del hombre y de la cultura. Cinco ensayos, introd. de G. Hoyos VÁsquez, trad. y notas de A. SeRRANo de HaRo, Barcelona/México: Anthropos/Universidad Autónoma Metropolitana, Iztapalapa, (orig. «Fünf Aufsätze über Erneuerung», en Aufsätze und Vorträge (1922-1937), Hua XXVII, 3-94.

- (2004), Meditaciones cartesianas. Trad. de José GaOs y M. García-Baró. México DF: FCE.

IRIBARNE, Julia V. (1987a), "La fenomenología como monadología", en Escritos de Filosofía, 15-16. Ahora en IRIBARne 2002, pp. 71-85.

- (1987b), La intersubjetividad en Husserl. Bosquejo de una teoría I. Buenos Aires: Carlos Lohlé.

- (1988), La intersubjetividad en Husserl. Bosquejo de una teoría II. Buenos Aires: Carlos Lohlé.

- (1990), "Interpretación ética de la 'interpretación' fenomenológica", en Cuadernos de Ética, 8, 33-34. Actualmente en IRIBARne 2002, cap. XVII. 
- (1992), "El concepto husserliano de mónada", en Escritos de Filosofía, 2122. Ahora en Iribarne 2002, pp. 87-102.

- (2002), Edmund Husserl. La fenomenología como monadología. Buenos Aires: Academia Nacional de Ciencias de Buenos Aires.

- (2005), "Acerca de los avatares del llegar a ser 'uno' mismo", en http://www.ciencias.org.ar/user/FILE/IRIBARNE.pdf (consultado 2014).

- (2007), De la ética a la metafísica. En la perspectiva del pensamiento de Edmund Husserl. Bogotá: Sociedad San Pablo-Universidad Pedagógica Nacional.

- (2008), "Ética y antropología", cap. IX en A. RoCHA DE LA TORRE 2008, pp. 66-107.

- (2009), "Estudio preliminar" a Husserl y lo político, trad. de K. SCHUHMANN, Husserls Staatsphilosophie. Buenos Aires: Prometeo Libros, pp. 15-30.

ORTH,Wolfgang (1977): "Anthropologie und Intersubjektivität. Zur Frage von Transzendentalität oder Phänomenalität der Kommunikation", en Phänomenologische Forschungen, 4, 103-129.

ROCHA DE LA TORRE, Alfredo (ed.) (2008), La responsabilidad del pensar, Barranquilla (Colombia): Ediciones Uninorte.

SAN MARTín, J. (1991), "Phénoménologie et Anthropologie", en Études phénoménologiques 13-14, 85-114. Traducido como cap. V en SAN MARTín, 1994, pp. 165-196.

- (1994), La fenomenología como teoría de una racionalidad fuerte, Madrid, UNED.

- (1995), "Husserl y la antropología cultural. Comentario a La carta de Husserl a Lévy-Bruhl", en ER, Revista de Filosofía, 19, año X, pp. 177-204. Actualmente en SAN MARTÍN, 2007, 125-152.

- (2007), Para una filosofía de Europa. Ensayos de fenomenología de la historia. Madrid: Biblioteca Nueva/UNED.

- (2012), "En torno a la nueva visión de Husserl", Valenciana 9, (Guanajuato, México), 2012. pp. 182-206.

- (2015), La nueva imagen de Husserl. Lecciones de Guanajuato, Madrid: Editorial Trotta.

Schummann, Karl (1977), Husserl-Chronik. Denk- und Lebensweg Edmund Husserls, La Haya: Martinus Nijhoff.

SEPP, Hans Rainer (1994), „Husserl über Erneuerung. Ethik im Schnittfeld von Wissenschaft und Sozialität", en Gerlach, H.-M- u. SePP, H. R. (eds.), Husserl in Halle. Spurensuche im Anfang der Phänomenologie. Frankfurt a. M.: Peter Lang, 109-130. 
- (1997), Praxis und Theorie. Husserls transzendentalphänomenologische Rekonstruktion des Lebens. Freiburg/München: Karl Alber.

WALTON, Roberto (2002), "Prólogo" a Iribarne 2002, pp. 9-22.

- (2005): "Discurso de recepción [de la académica J. V. Iribarne] del Académico Titular Dr. Roberto J. Walton", en http://www.ciencias.org.ar/user/FILE/IRIBARNE.pdf, (consultado en 2014).

Welton, Don (2003), The new Husserl. A Critical Reader, Bloomington: Indiana University Press. 